\title{
A New Approach to Prediction of Fatigue Life for 2024-T42 Aluminum under Variable Amplitude Loading
}

\author{
Yu-Bo GUOa, Ke-Ge LIU, Le LIU and Zhen TAO \\ Beijing Aircraft Strength Institute, Beijing, 100083, P.R. China
}

Keywords: Life prediction, Fatigue damage, variable amplitude loading, 2024-T42 aluminum.

\begin{abstract}
A new approach is explored to predict the fatigue life of 2024-T42 aluminum subjected to variable amplitude loading. The predicted fatigue lives are compared with the experimental data and those predicted by Miner's rule, which show that the fatigue lives predicted by the proposed approach agree well with the experimental results and most of the errors are within a factor of 2 . The proposed approach gives better prediction than the Miner's rule owing to the fact that it can take into account the loading history. Furthermore, the theoretical results tend to be non-conservative for the decreasing loading and the predicted results underestimate the experimental lives for the increasing loading.
\end{abstract}

\section{Introduction}

The aluminum alloy 2024-T42 is widely used in aerospace structure subjected to variable amplitude loading. So how to predict the fatigue life for aluminum alloy 2024-T42 under variable loading can be critical to ensure the safety and reliability of aerospace structure. The Miner's rule is the classical method to assess fatigue life of metal material sunder variable loading, which can be expressed as [1]:

$$
D=\sum_{i=1}^{n} \frac{n_{i}}{N_{R i}}=\sum_{i=1}^{n} r_{i}
$$

where $n_{i}$ and $N_{R i}$ is the consumed number of cycles and the number of cycles to failure in accordance with a stress amplitude $\Delta \sigma_{i}$ respectively. $\mathrm{D}$ is the damage variable and increasing with the fatigue damage accumulation until the fracture happens, then $\mathrm{D}=1$.

However, the Miner's rule will be detrimental to predict the fatigue life of material for its assessment error due to the lack of loading history. That is, the fatigue lives predicted by Miner rule are lower than experimental values the for increasing loading and are higher than the experimental results for decreasing loading under the same loading level[2]. Therefore, many methods [3-12] has been conducted to predict fatigue life under variable loading condition based on nonlinear damage cumulative model. Especially, the literatures [13-16] explore methods to predict the fatigue life of aluminum alloy 2024-T42 subjected to variable amplitude loading. Pavlou DG [13]discuss a phenomenological approach to estimating the fatigue life of 2024-T42 aluminum alloy in view of hardness change. Mesmacque $\mathrm{G}$ and A. Aid [14-15] propose a method to predict the fatigue life of 2024-T42 aluminum alloy based on Damage Stress Model. A. Djeblin[16] use a non-linear energy model to calculate the fatigue life of 2024-T42 aluminum alloy. The comparison between the predicted fatigue lives and the experimental results show that the proposed models present a good assessment of fatigue life for 2024-T42 aluminum alloy under variable amplitude loading condition.

The aim of this paper is to study an approach to predict the fatigue life for structure components made of 2024-T42 aluminum alloy subjected to variable amplitude loading.

\section{Description of New Approach}

The approach is presented on the basis of the non-linear energy model [16]. The hypothesis employed in this approach is that when the physical state of damage is unchanged, then the fatigue life will depend only on loading conditions [14]. Under high-cycle fatigue, the relationship between the energy and fatigue life can be written as follows[16]: 


$$
W_{a}=k\left(2 N_{f}\right)^{c^{\prime}}
$$

where $k=\left(\sigma_{f}^{\prime}\right)^{2} / 2 E$ and $c^{\prime}=2 b$.

A new damage parameter $D_{i}$ is defined as the ratio of the difference between energy due to the applied stress and the fatigue limit stress over the difference between energy due to the damage stress and the fatigue limit stress, which can be expressed as:

$$
D_{i}=\frac{W_{i}-W_{-1}}{W_{d s, i}-W_{-1}}
$$

where $W_{i}$ is the energy due to the applied stress, $W_{d s, i}$ is the energy due to the damage stress and $W_{-1}$ is the energy due to the fatigue limit stress. The damage stress is regarded as an admissible stress which corresponds to $n_{i}$ cycles of loading applied at level ion the Wöhler curve. The damage indicator $D_{i}$ is equal to one at rupture. Damage will be translated to level $i+1$ by the following relationship:

$$
D_{i}=\frac{W_{i}-W_{-1}}{W_{d s, i}-W_{-1}}=\frac{W_{i+1}-W_{-1}}{W_{d s e q, i+1}-W_{-1}}
$$

where $W_{\text {deeq, } i+1}$ and $W_{i+1}$ are the energy due to the damage equivalent stress and the damage stress at level $\mathrm{i}+1$, respectively.

This damage accumulation procedure will be continued until the damage variable $D=1$, then the damage stress is the same as the applied stress. In this way the loading history can be taken into account and the more accurate results are expected to be achieved.

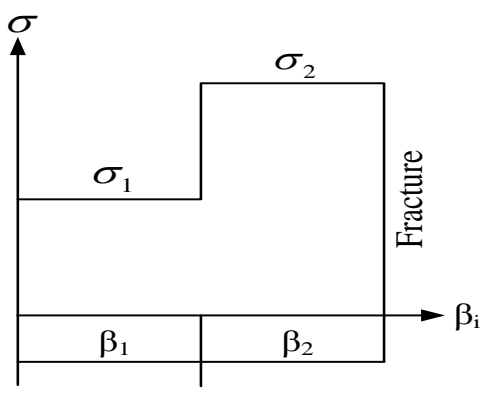

(a)

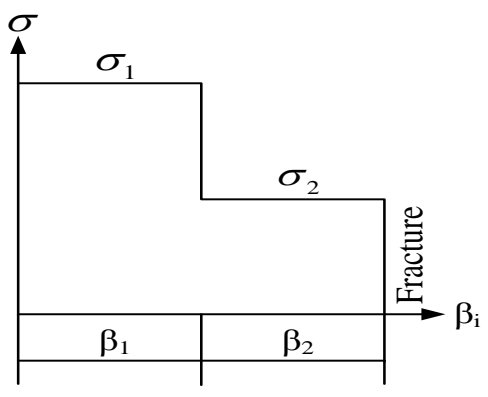

(b)

\begin{tabular}{|c|c|c|c|c|c|}
\hline \multicolumn{6}{|c|}{$\sigma_{1}=150 \mathrm{MPa}, \quad \sigma_{2}=200 \mathrm{MPa}$} \\
\hline \multirow[t]{2}{*}{$\mathrm{n}_{1}$} & \multicolumn{3}{|c|}{$\mathrm{n}_{2}$} & \multicolumn{2}{|c|}{ Relative error (\%) } \\
\hline & Exp. & Miner & Proposed approach & Miner & Proposed approach \\
\hline \multirow{3}{*}{86000} & 138,000 & \multirow{3}{*}{120000} & \multirow{3}{*}{129659} & 13.043 & -6.044 \\
\hline & 147,000 & & & 18.367 & -11.797 \\
\hline & 148,500 & & & 19.192 & -12.688 \\
\hline \multirow{3}{*}{172000} & 138,000 & \multirow{3}{*}{90000} & \multirow{3}{*}{103517} & 34.783 & -24.988 \\
\hline & 139,500 & & & 35.484 & -25.794 \\
\hline & 123,000 & & & 26.829 & -15.840 \\
\hline \multirow{3}{*}{258000} & 89,000 & \multirow{3}{*}{60000} & \multirow{3}{*}{73462} & 32.584 & -17.458 \\
\hline & 81,000 & & & 25.926 & -9.306 \\
\hline & 75,000 & & & 20.000 & -2.051 \\
\hline
\end{tabular}

Fig. 1 Two loading cycle blocks with: (a) increasing amplitude (b) decreasing amplitude.

Table.1 Experimental results, Miner and Proposed model predicted life for increasing loading 
Table.2 Experimental results, Miner and Proposed model predicted life for decreasing loading.

\begin{tabular}{|c|c|c|c|c|c|}
\hline \multicolumn{6}{|c|}{$\sigma_{1=} 200 M p a, \sigma_{2}=150 M p a$} \\
\hline \multirow[t]{2}{*}{$\mathrm{n}_{1}$} & \multicolumn{3}{|c|}{$\mathrm{n}_{2}$} & \multicolumn{2}{|c|}{ Relative error $(\%)$} \\
\hline & Exp. & Miner & Proposed approach & Miner & Proposed approach \\
\hline \multirow{3}{*}{30,000} & 259,100 & \multirow{3}{*}{344000} & \multirow{3}{*}{318491} & 32.767 & 22.922 \\
\hline & 233,400 & & & 47.386 & 36.457 \\
\hline & 193,500 & & & 77.778 & 64.595 \\
\hline \multirow{3}{*}{60,000} & 90,300 & \multirow{3}{*}{258000} & \multirow{3}{*}{225204} & 185.714 & 149.395 \\
\hline & 98,250 & & & 162.595 & 129.215 \\
\hline & 114,600 & & & 125.131 & 96.513 \\
\hline \multirow{3}{*}{90,000} & 86,000 & \multirow{3}{*}{172000} & \multirow{3}{*}{143527} & 100.000 & 66.892 \\
\hline & 42,300 & & & 306.619 & 239.307 \\
\hline & 99,800 & & & 72.345 & 43.815 \\
\hline
\end{tabular}

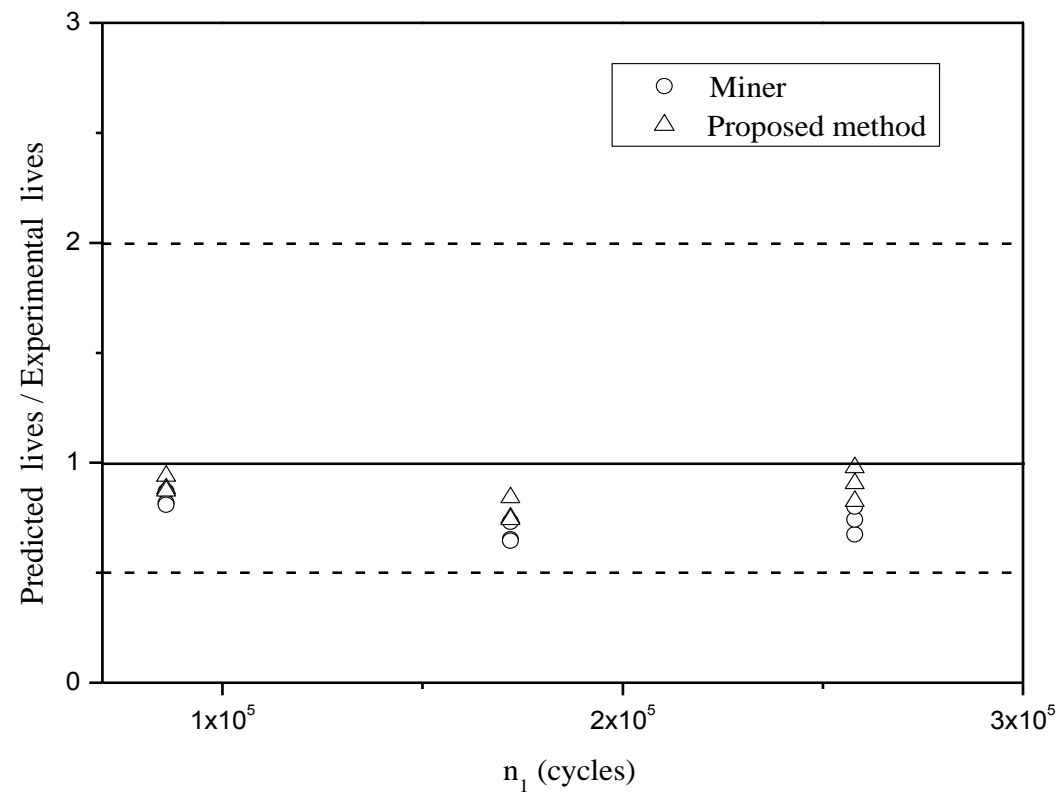

Fig.2 Comparison of experimental results, Miner and Proposed approach predicted fatigue lives under increasing loading. 


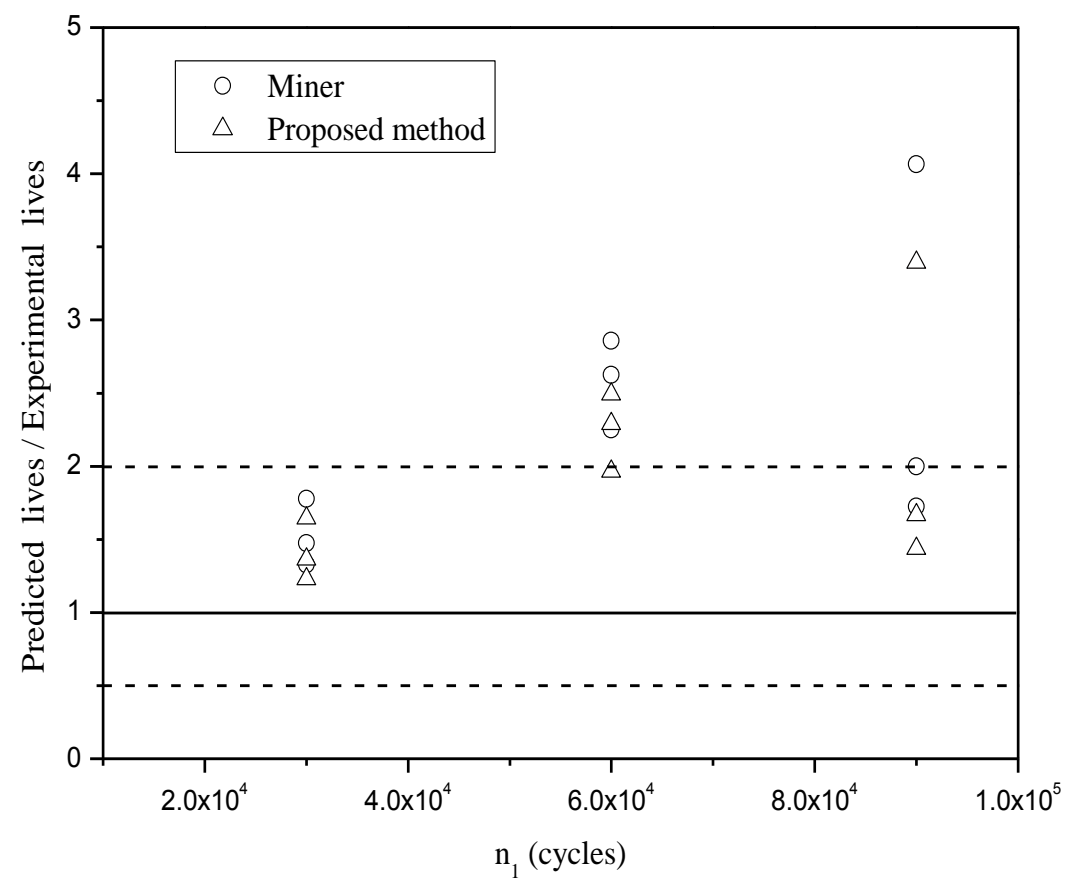

Fig.3 Comparison of experimental results, Miner and Proposed approach predicted fatigue lives under decreasing loading

\section{Life prediction by the Proposed Approach}

The material studied in this paper is the Aluminum alloy 2024-T42. The parameters used in the modified Wöhler curve are $\mathrm{k}=187$ and $\mathrm{c}=-0.546$. Lifetime for $\sigma=150 \mathrm{MPa}$ is $\mathrm{N}=430,000$ cycles, $\mathrm{W}=0.1563 \mathrm{MJm}^{-3}$ and for $\sigma=200 \mathrm{MPa}$ is $\mathrm{N}=150,000$ cycles, $\mathrm{W}=0.2778 \mathrm{MJm}^{-3} . \mathrm{W}_{-1}$ is the energy due to the fatigue life of $2 \times 10^{6}$ cycles, and $\mathrm{W}_{-1}=0.06783 \mathrm{MJm}^{-3}$. The loading conditions are illustrated in Fig.1, in which $\beta_{1}$ is the fraction of life at level $1, \beta_{2}$ is the fraction of life at level 2 until fracture.

The results predicted by the proposed approach are compared with the test data, which are listed in Tables 1 and 2. The comparison of experimental results, fatigue lives predicted by Miner's rule and the proposed approach is illustrated in Figures 2 and 3, in which the consumed cycles at stress level 1 is the ordinate and the ratio between the predicted lives and the experimental lives is the abscissa. It can be demonstrated that the predictions by the proposed approach in this paper are in encouraged agreement with the test results and most of the errors for the predicted lives are within a factor of 2 . Besides, it seems that the theoretical results tend to be non-conservative for the decreasing loading and conservative for the increasing loading. Furthermore, the fatigue lives predicted by the proposed approach have smaller error than those by Miner's rule, which will be attributed to the fact that the proposed approach can take into account the loading history.

\section{Conclusions}

In this paper the fatigue lives of 2024-T42 aluminum are predicted based on a new approach under variable amplitude loading, by which the loading history can be taken into account. The predicted lives are compared with the experimental and Miner's results. It shows that the predictions by the proposed approach are in good agreement with the test data and most of the errors are within a factor of 2.Furthermore, the deviation of fatigue lives predicted by the proposed approach is lower than those predicted by Miner's rule. In addition, it will be verified if the proposed approach can be extended to other materials and loading conditions in the further study. 


\section{Acknowledgements}

The authors gratefully acknowledge the financial support of the National Natural Science Foundation of China (Nos. 50135010).

\section{References}

[1] MA Miner. Cumulative damage in fatigue. J ApplyMech;67:A159-64(1945).

[2] A Fatemi, L Yang. Cumulative fatigue damage and life prediction theories: a survey of the state of the art for homogeneous materials. Int J Fatigue;20:9-34 (1998).

[3] ShangDe-Guang, Yao Wei-Xing.A nonlinear damage cumulative model for uniaxial fatigue. Int J Fatigue;21:187-194(1999).

[4] Y Jiang, W Ott, C Baum, M Vormwald, H Nowack. Fatigue life predictions byintegrating EVICD fatigue damage model and an advanced cyclic plasticitytheory. Int J Plasti;25:780-801(2009).

[5] RPL Nijssen, DRV Van Delft, AM Van Wingerde. Alternative fatigue lifetimeprediction formulations for variable-amplitude loading.J Sol Energy Eng;124(4396):396-403(2002).

[6] V Dattoma, S Giancane, R Nobile, FW Panella. Fatigue life prediction under variable loading based on a new nonlinear continuum damage mechanics model. Int J Fatigue; 28(2):89-95(2005).

[7] A Varvani-Farahani, M Sharma, MR Kianoush. Fatigue damage analysis and lifeassessment under variable amplitude loading conditions. Mater SciEng A;403:42-7(2005).

[8] G Pereira, P Jesus, AS Ribeiro, AA Fernandes. Fatigue damage behaviour ofstructural components under variable amplitude loading. MecExp;17:75-85(2009).

[9] S Mikheevskiy, G Glinka. Elastic-plastic fatigue crack growth analysis undervariable amplitude loading spectra. Int J Fatigue;31:1828-36(2009).

[10] S Oller, O Salomon, E Onate. A continuummechanics model formechanical fatigue analysis.Comput Mater Sci;32:175-95(2005).

[11] H. Jahed, A. Varvani-Farahani, M. Noban, I. Khalaji, An energy-based fatiguelife assessment model for various metallic materials under proportional andnon-proportional loading conditions, International Journal of Fatigue; 4:647-655(2007).

[12] HA Xiaoping, T Moan, C Weicheng. An engineering model of fatiguecrack growth under variable amplitude loading. Int J Fatigue;30:2-10(2008).

[13] DG Pavlov. A phenomenological fatigue damage accumulation rule based on hardness increasing, for the 2024-T42 aluminum. EngStruc;24:1363-8(2002).

[14] G Mesmacque, S Garcia, AAmrouche. Sequential law in multiaxial fatigue: a new damage indicator. Int J Fatigue;27(4):461-7(2005).

[15] A.Aid, A.Amrouche, B. BachirBouiadjra, M. Benguediab, G.Mesmacque. Fatigue life prediction under variable loading based on a new damage model. Mater. Des; 32:183-191(2011).

[16]A.Djebli, A.Aid, M.Bendouba,A.Amrouche, M.Benguediab, N.Benseddiq. A non-linear energy model of fatigue damage accumulation and its verification for Al-2024 aluminum alloy. Int J NonlinMech;51:145-151(2013). 